\title{
Klassen zusammenhängender Gruppen
}

\author{
Von \\ Pemer Plaumana
}

In [8] haben wir die in der Klasse 8 aller lokal kompakten Gruppen mit kompakter Zentrumafaktorgruppe bzw. in der Klasse $\mathfrak{D}$ aller lokal kompakten Gruppen mit kompakter Kommutatorgruppe enthaitenen zusammenhängenden Gruppen durch Angabe von äquivalenten Eigenschaften charakterisiert. Werm wir mit $\xi \mathfrak{X}$ die Klasse aller zusammenhängenden abgeschlossenen Untergruppen von Gruppen aus der Klasse $\mathfrak{X}$ lokal kompakter, zusammenhängender Gruppen bezeichnen, so erhalten wir folgende Kriterien dafür, daß eine Klasse $\mathrm{B}$ lokal kompakter, zusammenhängender Gruppen eine Teilkzasse von $\mathfrak{B}$ bzw, 8 ist:

genau dann ist $\mathfrak{x}$ eine Teilklasse von $\mathfrak{D}$, wem der Durchschnitt von $3 \mathfrak{A}$ mit der Klasse aller auflösbaren Gruppen in der Klasse aller nilpotenten Gruppen der Klasse 2 liegt und der Durchschnitt von $3 \mathfrak{s}$ mit der Klasse der einfach zusammenhängenden auflösbaren Liegruppen in der Klasse aller abelschen Gruppen liegt (Korollar 2), Genau dann ist $\mathfrak{R}$ in der Klasse $B$ enthalten, wenn der Durchsobnitt von $3 \mathfrak{S}$ mit der Klasse aller auflösbaren Gruppen in der Klasse aller abelschen Gruppen enthalten ist (Korollar 1).

Den Schlüssel zum Beweis dieser Aussagen bildet der Satz 1, der die Teilklassen der Klasse $\widetilde{F}$ aller lokal kompakten, zusammenhängenden Gruppen charakterisiert, in denen das Schlußglied der unteren Zentralreihe kompakt ist:

Die Klasse $\mathfrak{E}$ ist genau dann Teilklasse von $\mathfrak{F}$, wenn der Durchschnitt von $\mathfrak{S} \mathfrak{B}$ mit der Klasse aller auflösbaren Gruppen in der Klasse der nilpotenten Gruppen enthalten ist. Weiterhin enthält Satz 1 eine Strukturaussage über die Gruppen aus $\Im$, die es gestattet, den Satz von IWASAwa und Schmid, daB eine endliche Gruppe, deren auflösbare Untergruppen nilpotent sind, selbst nilpotent ist, auf die Klasse der lokal kompakten Gruppen, die endlich über der zusammenhängenden Komponente der Ains sind, zu erweitern.

Die in der vorliegenden Note gewählten Bezeichnungen sind zum größten Teil Standard, stimmen aber auf jeden Fall mit den Bezeichnungen aus [8] überein.

Zunächst beweisen wir einige Hilfssätze.

Hilissatz 1. Die untere Zentralreihe

$$
G=G_{1} \supseteqq \ldots \supseteqq G_{i} \supseteqq Q_{i+1}=D\left(G, G_{i} \supseteqq \ldots \supseteqq G_{\mu}=\bigcap_{\lambda<\mu} G_{\lambda} \supseteq G_{x}=D\left(G, G_{\tau}\right)\right.
$$

einer lokal kompahten, zusammenhängenden Gruppe $\mathrm{Q}$ ist endich. 
Beweis. Der Beweis verläuft analog zu dem in [6] für Theorem 5 angegebenen, wenn man Lemma 2.2 durch folgende Aussage ersetzt:

Sei $G$ eine lokal kompalte, zusammenhängende Gruppe mil kompaktem $G_{k}$. Dann ist $G_{k+1}=G_{k+2}$.

Wir beweisen diese Aussage. Nach [6], Lemma 2.4, ist $G_{k}=\left(Z G_{k}\right)_{0}\left(D G_{k}\right)$. Da $\left(Z G_{k}\right)_{0}$ wegen [6], Theorem 4 , in $Z G$ enthalten ist, gilt

und

$$
G_{k+1}=D\left(G, G_{k}\right)=D\left(G, D G_{k}\right)=D G_{k}
$$

$$
G_{k+2}=D\left(G, G_{k+1}\right)=D\left(G, D G_{k}\right)
$$

Hilissatz 2. In einer kompakten, zusammenhängenden Gruppe $G$ ist jedes Element in einer zusammenhängenden abelschen Untergruppe enthalten.

Beweis. Nach [10], §25, hat $G$ folgende Struktur:

$$
G=\left(A \times P_{\gamma \in I}, L_{\gamma}\right) / D \text {. }
$$

Dabei ist $A$ eine kompakte, zusammenhängende abelsche Gruppe, die Gruppen $L_{\gamma}$ sind kompakte einfache Liegruppen, und $D$ ist ein total unzusammenhängender Normalteiler von $A \times P_{\gamma \in T} L_{\gamma}$. Sei nun $x \in G$, und sei $y$ ein Repräsentant von $x$ in $A \times P_{\gamma \in \Gamma} L_{\gamma}$. Mit $\pi_{\gamma}$ bezeichnen wir die kanonische Projektion von $A \times P_{\gamma \in \Gamma} L_{\gamma}$ auf $L_{\gamma}$. Wegen [4], Nr. 17, gibt es in $L_{\gamma}$ eine zusammenhängende abelsche Untergruppe $T_{\gamma}$ mit $y \pi_{\gamma} \in T_{\gamma}$. Dann ist $\left(A \times P_{\gamma \in I} T_{\gamma}\right) D / D$ die gesuchte Untergruppe von $G$.

Hilfssatz 3. Sei $G$ eine lokal kompakte, zusammenhängende Gruppe, und sei $R$ das auflösbare Radikal von $G$. Wenn es in $G$ einen kompakten Normalteiler $K$ mit au ${ }^{-}$ lösbarem $G / K$ gibt, so ist $G=R K$.

Beweis. Die Gruppe $G / R K$ ist als epimorphes Bild von $G / R$ halbeinfach und als epimorphes Bild von $G / K$ auflösbar.

Satz 1. Für eine lokal kompakte, zusammenhängende Gruppe $G$ sind die folgenden Aussagen äquivalent:

(1) Alle zusammenhängenden auflösbaren Untergruppen von G sind nilpotent.

(2) In $Q$ gibt es einen nilpotenten Normalteiler $N$ und einen kompakten halbeinfachen Normalteiler $K$, der total unzusammenhängenden Durchschnitt mit $N$ hat, so daß $G=N K$ ist.

(3) Das Schlußglied der unteren Zentralreihe von $G$ ist kompalt.

(4) Q ist die Erweiterung einer kompakten Gruppe durch eine nilpotente Gruppe.

Beweis. Es gelte zunächst (1), und es sei $R$ das auflösbare Radikal von $G$. Wir wollen zeigen, daß der halbeinfache Kopf $H=G / R$ von $G$ kompakt ist. Wegen der Auflösbarkeit von $R$ erfüllt auch $H$ die Bedingung (1). Sei $C$ ein kompakter Normalteiler von $H$ mit Liescher Faktorgruppe $H / C$, und sei $U / C$ eine zusammenhängende auflösbare Untergruppe von $H / C$. Wegen Hilfssatz 3 ist $U=S C$, wobei $S$ das auflösbare Radikal von $U$ ist. Aus der Nilpotenz von $S$ folgt dann die Nilpotenz von $U / C$. Da $Z(H / C)$ diskret ist, sieht man leicht ein, daß auch ad $H / C \cong(H / C) / Z(H / C)$ die 
Bedingung (1) erfüllt. Da die Kompaktheit von $H / C$ zur Kompaktheit von ad $H / C$ gleichwertig ist, können wir also o.B.d.A. annehmen, da. $Z(H \mid C)=1$ ist. Nach $[1], 15.5$, hat dann $H / C$ folgende Struktur: Es gibt eine auflösbare, einfach zusammenhängende Untergruppe $A$ und eine kompakte, zusammenhängende Untergruppe $B$, so da $B H / C=A B$ ist. Da $H / C$ der Voraussetzung (1) genügt, ist $A$ nilpotent. Da der Restklassenraum $(H / C): A$ kompakt ist, folgt aus $[2]$, Satz B, daß $H / C$ über seinem maximalen zusammenhängenden nilpotenten Normalteiler $N(H / C)$ kompakt ist. Die Halbeinfachbeit von $H / C$ hat $N(H / C)=1$ und damit die Kompaktheit von $H / C$ zur Folge. Da $H$ projektiver Limes von Liegruppen ist (vgl. [7], Theorem 4.6), folgt die Iompaktheit von $H=G / R$. Nun ist $R$ nilpotent. Also gibt es nach dem Hauptsatz aus [2] eine kompakte, zusammenhängende halbeinfache Untergruppe $K$ von $G$, deren Durchschnitt mit $R$ total unzusammenhängend ist, so da $B G=R K$ ist. Wir haben nun noch zu zeigen, daß $K$ ein Normalteiler von $G$ ist. Sei $x \in K$ und sei $T$ eine zusammenhängende abelsche Untergruppe von $K$ mit $x \in T$ (s. Hilfssatz 2). Die Untergruppe $R T$ von $G$ ist auflösbar, also nilpotent. Daraus folgt $T \subseteq Z(R T$ ) (s. etwa [8], Satz 2.1). Also ist $D(R, T\rangle=1$, und es folgt

$$
D(G, K)=D(R K, K)=D(R, K) D K=D K \leqq K .
$$

Nun gelte (2), und es sei $n$ die Nilpotenzklasse von $N$. Dann ist $G_{n+1}$ in $K$ enthalten und damit kompakt.

Da nach Hilfssatz 1 die untere Zentralreihe von $G$ nach endlich vielen Schritten abbricht, folgt (4) aus (3).

Sohließlich gelte (4), und es sei $U$ eine zusammenhängende auflösbare Untergruppe von $Q$. Wenn $K$ ein kompakter Normalteiler von $G$ mit nilpotentern $Q / K$ ist, so ist auch $U /(U \cap K)_{0}$ nilpotent. Nach [6], Lemma 2.2 und Theorem 4 , ist $(U \cap K)_{0}$ in $Z U$ enthalten, so daß $U / Z U$ und damit $U$ nilpotent ist.

Korollar 1. Sei $G$ eine lokal kompakte, zusammenhängende Gruppe. Dann sind die tolgenden Aussagen äguivalent:

(1) Alle aullösbaren zusammenhängenden Untergruppen von $Q$ sind abelsch.

(2) $Q$ ist das direkte Produkt einer Vektorgruppe und einer kompakten Gruppe.

(3) G ist eine B-Gruppe [Die Zentrumsfaktorgruppe von $Q$ ist kompakt.]. Beweis. Wenn (1) gilt, so ist $Q$ nach Satz 1 das Produkt eines nilpotenten Normal-
teilers $N$ und eines kompakten Normalteilers $K$. Da $N$ nach Voraussetzung abelsch
ist, folgt ist, folgt daraus (2). Daß (3) aus (2) folgt, ist trivial. Nach [8], Bemerkung zu Satz 4.2, unitgenau dann eine B-Gruppe, wenn es in $G$ hinreichend viele endlich-dimensionale unitäre Darstellungen gibt. Also sind mit $G$ anch alle zusammenhängenden Untergruppen 3-Gruppen. Wegen [8], Satz 4.2 ist jede 3-Gruppe das direkte Produkt oiner Vektorgruppe und einer kompakten Gruppe. Daraus folgt, daß auflösbare zusammenhängende 8 -Gruppen abelsch sind. Also folgt (1) aus (3).

Korollar 2. Für eine lokal kompakte, zusammenhüngende Gruppe G sind die folgenden Aussagen äquivalent:

(1) a) Die zusammenhängenden auflösbaren Untergruppen von $G$ sind nilpotent der Klasse höchstens 2. 
b) Die einfach zusammenhängenden nilpotenten Untergruppen von $G$ sind abelsch. (2) $G$ ist das Produkt eines nilpolenten $D$-Normalteilers $N$ und eines kompakten halbeinfachen Normalteilers $K$.

(3) G ist eine D-Gruppe [Die Kommutatorgruppe von $G$ ist kompakt.].

Beweis. Aus (1.a) folgt nach Satz 1 die Existenz eines nilpotenten Normalteilers $N$ und eines kompakten halbeinfachen Normalteilers $K$, so daß $G=N K$ ist. Wir haben zu zeigen, daB $N$ eine $D$-Gruppe ist. Seien dazu $R$ und $V$ zu $\mathbf{R}$ isomorphe Untergruppen von $N$. Da $N$ nilpotent der Klasse 2 ist, wird für jedes $r \in R$ durch

$$
\varrho: v \rightarrow[r, v]
$$

ein Homomorphismus von $V$ in $N$ definiert. Also ist entweder $D(V, r)=\bar{V} \varrho$ kompalst oder es ist $V^{e}=\bar{V} \varrho \cong \mathbf{R}$. Angenommen, letzteres wäre der Fall. Da für alle $v \in V$

$$
\begin{aligned}
{\left[r^{1 / 2}, v\right] } & =\left[r^{1 / 2},\left(v^{1 / 2}\right)^{2}\right]=\left[r^{1 / 2}, v^{1 / 2}\right]^{2}= \\
& =\left[r, v^{1 / 2}\right]=\left(v^{1 / 2}\right)^{\varrho} \in V \varrho
\end{aligned}
$$

ist, wäre für die dichte Untergruppe $U=\left\{r^{2^{k}} ; k\right.$ ganz $\}$ von $R$ die Beziehung $D(U, V)=V \varrho$ orfüllt. Also wäre auch $D(R, V)=V \varrho \cong \mathbf{R}$. Dann wären aber $R$ und $V$ in einer einfach zusammenhängenden Untergruppe von $G$ enthalten, die nach Voraussetzung abelsch wäre. Also wäre $D(R, V)=1$, q.e.a. Es folgt, daß für alle $r \in R$ die Gruppe $\bar{V} \varrho=D(r, V)$ kompakt ist. Da $N$ eine eindeutig bestimmte max $i$ male kompakte Untergruppe besitzt, ist dann auch $D(R, V)$ kompakt. Nach Theorem 13 aus [6] gibt es in $N$ eine kompakte Untergruppe $C$ und zu $\mathbf{R}$ isomorphe Untergruppen $R_{i}, i=1, \ldots, n$, so daß $N=R_{1} \cdots R_{n} C$ ist. Also ist $D N=\prod_{i, j} D\left(R_{i}, R_{j}\right)$
kompakt.

Wenn (2) gilt, so folgt (3) aus der Beziehung $D G=(D N)(D K)$.

Schließlich gelte (3). Da Untergruppen von $\mathfrak{D}$-Gruppen wieder $\mathcal{D}$-Gruppen sind, and da auflösbare zusammenhängende $\mathfrak{D}$-Gruppen wegen [6], Lemma 2.2 und Theorem 4, nilpotent der Klasse 2 sind, folgt zunächst (1. a). Aus der Tatsache, da $\beta$ eine einfach zusammenhängende nilpotente Gruppe kompaktfrei ist, folgt dann auch (1. b).

Satz 2. Eine lokal kompakte Gruppe $G$ mit endlichem $G / G_{0}$ ist genau dann nilpotent, wenn ihre auflösbaren Untergruppen nilpotent sind.

Beweis. Nach Satz 1 gibt es in $G_{0}$ einen nilpotenten Normalteiler $N$ und einen kompakten halbeinfachen Normalteiler $K$, so daß $G_{0}=N K$ ist. Sei $L$ ein Liescher Normalteiler von $K$, sei $T$ ein maximaler Torus von $L$ (s. [4], Nr. 23), sei $p \neq 2$ eine Primzahl, und sei $P=\left[x \in T ; x^{p}=1\right]$. Da $C_{L} T=T$ ist (s. [4], l.c.), ist $N_{L} T / T$ eine Gruppe von Automorphismen von $T$. Wäre nun $L \neq 1$, so gäbe es nach [9], Satz 12 und Nr. 10, eine Involution $\alpha$ in $N_{L} T / T$. Da $P$ eine charakteristische Untergruppe von $T^{\prime}$ ist, wäre $P^{\alpha}=P$. Sei $a$ ein Repräsentant von $\alpha$ in $N_{L} T$. Dann wäre $P\{a\}$ eine auflösbare Untergruppe von $Q$ und damit nilpotent. Mit der Definition

$$
\left[x, \xi^{(0)}\right]=x, \ldots,\left[x, \xi^{(i+1)}\right]=\left[x, \xi^{(i)}\right]^{\xi}\left[x, \xi^{(i)}\right]^{-1},
$$

für $\xi=a$ oder $\xi=\alpha$, gäbe es dann eine natürliche Zahl $n$, so daß für alle $x \in P$ diø 
Engel-Bedingung

$$
\left[x, \alpha^{(n)}\right]=\left[x, a^{(n)}\right]=1
$$

erfüllt wäre. Also wäre das semidirekte Produkt von $P$ und $\{\alpha\}$ eine endliche nilpotente Gruppe. Wegen $p \neq 2$ würde daraus folgen, daß $P$ von $\alpha$ zentralisiert wird. Also würde $\alpha$ auch die dichte Untergruppe $Q=\left[x \in T ; x^{p^{k}}=1, k\right.$ ganz $]$ von $T$ zentralisieren, q.e.a. Aus diesem Widerspruch folgt $L=1$ und damit $K=1$, so daß $\sigma_{0}=N$ nilpotent ist.

Sei $U / G_{0}$ eine auflösbare Untergruppe der endlichen Gruppe $G / G_{0}$. Da mit $G_{0}$ auch $U$ auflösbar ist, folgt, daß $U$ und damit $U / G_{0}$ nilpotent ist. Nach dem Satz von $\mathrm{IW}_{\text {ASW }}$ und SchmIDT (s. [5], Kap. III, Satz 5.1) ist also $G / G_{0}$ nilpotent. Daraus folgt, da $B G$ auflösbar und somit nilpotent ist. Die Umkehrung ist trivial.

Korollar 3. Eine lokal kompakte Gruppe mit endlichem $G / G_{0}$ ist genau dann abelsch, wenn ihre auflösbaren Untergruppen abelsch sind.

Beweis. Wenn alle auflösbaren Untergruppen von $G$ abelsch sind, so ist $G$ nach Satz 2 selbst auflösbar.

\section{Literaturverzeichnis}

[1] K. H. Hormans, Lie Gruppen II. Vorlesung, Univ. Tübingen 1963.

[2] K. H. HofManN, Uber das Nilradikal lokal kompakter Gruppen. Math. Z. 91, 206-215 (1966).

[3] K. H. Hofmann and P. M. Mosterrt, Splitting in topological groups. Providence 1963.

[4] H. Hopr, Uber den Rang geschlossener Liescher Gruppen. Comm. Math. Helvet. 13, 119-143 $(1940)$.

[5] B. HUPPERT, Endliche Gruppen I. Berlin-Göttingen-Heidelberg 1967.

[6] K. IWASAWA, Some types of topological groups. Ann. of Math., II. Ser. 50, 507-558 (1949).

[7] D. MonTGOMERY and L. ZIPPIN, Topological transformation groups. New York 1955.

[8] P. PlaumanN, Erweiterungen kompakter Gruppen durch abelsche Gruppen. Math. Z. 99, $123-140(1967)$.

[9] E. STIEFTi, Utber eine Beziehung zwischen geschlossenen Lieschen Gruppen und diskontinuierlichen Bewegungsgruppen. Comm. Math. Helvet. 14, 350-380 (1942).

[10] A. W EIr, L'intégration dans les groupes topologiques. Paris 1951.

Anschrift des Autors:

Eingegangen am 29.11. 1966 ${ }^{1}$ )

Peter Plaumann

Department of Mathematics

Ann

Ann Arbor (Mich.), USA

1) Anmerkung der Redaktion: Eine erweiterte Fassung ging am 19. 7. 1967 ein. 